\title{
UGT1A1*28 polymorphism and acute lymphoblastic leukemia in children: a Danish case-control study
}

\author{
Jesper P. Petersen 1,2, Kim Overvad ${ }^{3}$, Mads V. Hollegaard ${ }^{4}$, Finn Ebbesen², Tine B. Henriksen' ${ }^{1}$, Ole Thorlacius-Ussing ${ }^{5}$, \\ David M. Hougaard ${ }^{4}$ and Henrik Schrøder ${ }^{1}$
}

\begin{abstract}
BACKGROUND: Oxidative stress is a possible risk factor in the development of acute lymphoblastic leukemia (ALL) in children. Bilirubin is a potent endogenous antioxidant, and the UGT1A $1 * 28$ polymorphism is the main genetic cause of variation in plasma bilirubin in Western Europe.
\end{abstract}

METHODS: In a case-control study of 665 incident cases of ALL in childhood in Denmark 1982-2010 and 1,379 controls, associations between UGT1A $1 * 28$ genotypes and ALL in childhood were estimated as odds ratios by logistic regression with adjustment for sex and birth decade. Subgroup analyses were carried out by age at onset in three groups, and on the ALL subtypes precursor B-cell, T-cell, and t(12;21) positive status. Cases were identified in The Danish Registry of Childhood Cancer, and genotypes were estimated from dried blood spots stored in The Danish Neonatal Screening Biobank. Controls were newborns with blood spots taken right before and after a case.

RESULTS: We found no association between ALL in childhood and UGT1 A $1 * 28$ genotypes. The odds ratio was 1.01 (0.88-1.17) for heterozygotes and $1.03(0.78-1.36)$ for homozygotes. Also, no associations were found in the subgroup analyses.

CONCLUSION: We found no association between the UGT1A1*28 genotypes and ALL in children.

D evelopment of acute lymphoblastic leukemia (ALL) in children is believed to involve at least two genetic "hits." The nature of these presumed "hits" and the factors associated with them have not yet been adequately described, but the first "hit" is assumed to often take place in utero (1). Furthermore, sex, birth weight, and birth order have been suggested as risk factors $(2,3)$. A possible risk factor for the development of ALL is oxidative stress because previous studies have shown a relationship between neonatal oxygen supplementation and later development of ALL $(4,5)$.

Bilirubin is a potent endogenous antioxidant with documented antioxidative effects in vitro and in vivo $(6,7)$. The possible clinical relevance of bilirubins antioxidative effects has been explored in adults, were observational studies of pulmonary function, cardiovascular disease, cancer and allcause mortality have indicated protective associations (8-10). In vitro studies, observational data and a single interventional trial suggest that bilirubin may be particularly important as an antioxidant in the neonatal period and in the preterm population. However, outside the first months of life bilirubin as an antioxidant has not been investigated in the pediatric population (11-13).

A challenge to the interpretation of observational studies on bilirubin is the possibility of reverse causation and potential confounding by sex, body mass index, diet, and smoking $(14,15)$.

A way of addressing possible confounding and reverse causality in observational studies is by employing genes as instrument variables for the phenotype of interest (Mendelian randomization) (16). If a genotype has a well-characterized relationship with a phenotype, this can be utilized to estimate the effect of the phenotype upon an exposure of interest. As the genotype is assigned randomly from parental genotypes at birth the estimate will be un-confounded by external factors and reverse causality will not be an issue (16). For bilirubin, such a study design is possible utilizing a common genetic variant-the UGT1 A1 ${ }^{*} 28$ allele.

The rate limiting enzyme in the metabolism of bilirubin is hepatic uridine diphosphate glucuronosyltransferase $1 \mathrm{~A} 1$ (UGT1A1). In the common genetic variant, the UGT1A1*28 allele, a thymine-adenine base pair has been inserted into the TATAA box of the promoter region of the UGT1A1 gene, resulting in seven TA pairs instead of the usual six. The TATAA box controls expression of the UGT1A1 gene, and UGT1A $1{ }^{\star} 28$ reduces the expression of hepatic UGT1A1 in a risk allele doseresponse manner. UGT1 $\mathrm{Al}^{\star} 28$ is the genotype behind GilbertMeulengracht's syndrome and the main known genetic cause of variations in plasma bilirubin in Western European populations accounting for $\sim 18 \%$ of all variations $(17-21)$. The allele frequency and high correlation to plasma bilirubin variations make UGT1A $1{ }^{\star} 28$ a possible instrument variable for bilirubin. This has been used in previous studies on the possible importance of bilirubin in association with cardiovascular disease, inflammatory bowel disease and colorectal cancer $(18,22,23)$. 
Hence, we investigated the relation between UGT1A1*28 genotypes and the development of ALL in children in a casecontrol study, encompassing incident cases in Denmark 1982-2010.

\section{RESULTS}

Between 1982 and 2010, 903 incident cases of ALL in children $1-14$ y old were present in the two databases; 679 could be identified in the biobank and had a dried blood spot deemed suitable for further analysis. For 14 of these, either DNA amplification or genotyping failed, making genotypes for 665 cases available for the study. In all, 1,419 controls had a blood spot drawn from the biobank for the study. In 40 of these, DNA amplification or genotyping failed; consequently a genotype was available in 1,379 controls. Baseline characteristics of cases and controls are shown in Table $\mathbf{1 .}$

Table 1. Baseline clinical characteristics for controls $(N=1,379)$ and cases $(N=665)$ (column statistics)

\begin{tabular}{lccc}
\hline Characteristic & & Controls & Cases \\
\hline Sex & Boys & $51.5 \%(710)$ & $56.4 \%(375)$ \\
Birth decade & Girls & $48.5 \%(669)$ & $43.6 \%(290)$ \\
& $1981-1989$ & $34.5 \%(476)$ & $34.4 \%(229)$ \\
ALL type & $1990-1999$ & $41.0 \%(565)$ & $40.8 \%(271)$ \\
& $2000-2009$ & $24.5 \%(338)$ & $24.8 \%(165)$ \\
Tge at onset & Pre-B-cell ALL & - & $12.3 \%(82)$ \\
& t(12;21) positive & - & $86.3 \%(574)$ \\
& $1-4 \mathrm{y}$ & - & $61.5 \%(409)$ \\
& $5-9 y$ & - & $25.9 \%(172)$ \\
& $10-14 \mathrm{y}$ & - & $12.6 \%(84)$ \\
\hline
\end{tabular}

Table 2. Sex and birth decade adjusted odds ratios (95\% confidence intervals) of acute lymphoblastic leukemia in children for UGT1A $1{ }^{*} 28$ allele genotypes

\begin{tabular}{lcccc}
\hline Genotype & $\begin{array}{c}\text { Controls } \\
(n=1,379)\end{array}$ & $\begin{array}{c}\text { Cases } \\
(n=665)\end{array}$ & OR $^{\mathrm{a}}$ & $95 \% \mathrm{Cl}$ \\
\hline $6 / 6$ & $658(47.7 \%)$ & $320(48.1 \%)$ & Ref. & Ref. \\
$7 / 6$ & $589(42.7 \%)$ & $276(41.5 \%)$ & 1.01 & $(0.87-1.16)$ \\
$7 / 7$ & $132(9.6 \%)$ & $69(10.4 \%)$ & 1.01 & $(0.77-1.34)$ \\
\hline UGT1A1*28 allele is referred to as 7, common genotype as 6. \\
\multicolumn{4}{l}{ Adjusted for sex and birth decade. }
\end{tabular}

The genotypes amongst the controls were in Hardy-Weinberg equilibrium $(P=0.94)$.

No association was found between ALL and UGT1A1*28 genotype. Using participants with the common genotype as a reference group, the odds ratio (OR) of ALL per UGT1A ${ }^{\star} 28$ allele was $1.01(0.88-1.17)$, making the OR for heterozygotes $1.01(0.88-1.17)$ and $1.02(0.77-1.36)$ for homozygotes. Treating genotype as a categorical variable gave similar estimates (data not shown). There was no evidence of an effect modification by sex or birth decade (data not shown), and adjusting for these by logistic regression left point estimates and confidence intervals almost unchanged. Fully adjusted ORs of ALL in childhood for genotypes are presented in Table 2.

For the pre-B-cell ALL, the T-cell ALL, the $\mathrm{t}(12 ; 21)$ translocation positive, and age at onset in the predefined intervals no association between genotype and outcome was found for unadjusted and adjusted estimates. Fully adjusted ORs for secondary outcomes are presented in Tables 3 and 4.

\section{DISCUSSION}

In this study, the UGT1A1*28 genotype was not associated with the risk of ALL in childhood.

The UGT1A1*28 genotype has drawn considerable attention in adult studies in recent years, primarily because of its influence on plasma bilirubin levels and the possibly associated increased antioxidative capacity (24). That the genotype is not associated with risk of ALL in children points toward bilirubin not having a relevant protective effect against this disease. However, some studies indicate that in the first days of life, UGT1A1 enzyme activity is very low, and correspondingly UGT1A $1{ }^{\star} 28$ genotype seems to have limited effect on serum levels of bilirubin at this age (25-28). Thus, if oxygen is primarily a risk factor for childhood ALL in the first minutes of life and/or in levels above atmospheric pressure, examining allele status will not be a relevant test of bilirubin's potential protective effect. A recent Danish register-based study with Apgar score as exposure does seem to suggest that the first minutes of life could be a particularly vulnerable period for the later development of some childhood cancers (29). Umbilical cord concentrations of bilirubin may be a more relevant measure of bilirubin exposure during the first days of life (30).

A main strength of our study is the completeness and nationwide coverage. Furthermore, the existence of a unique biological resource, the Danish Neonatal Screening Biobank, gave us

Table 3. Sex and birth decade adjusted odds ratios ( $95 \%$ confidence intervals) of acute lymphoblastic leukemia with age at onset in the intervals $1-4 y, 5-9 y$, and $10-14$ y for UGT1A $1 * 28$ genotypes

\begin{tabular}{|c|c|c|c|c|c|c|c|}
\hline \multirow[b]{2}{*}{ Genotype } & \multirow{2}{*}{$\begin{array}{l}\text { Controls } \\
(n=1,379)\end{array}$} & \multicolumn{2}{|c|}{ Age at ALL onset $1-4$ y $(n=409)$} & \multicolumn{2}{|c|}{ Age at ALL onset $5-9$ y $(n=172)$} & \multicolumn{2}{|c|}{ Age at ALL onset $10-14$ y $(n=84)$} \\
\hline & & & $\mathrm{OR}^{\mathrm{a}}$ & & $\mathrm{OR}^{\mathrm{a}}$ & & $\mathrm{OR}^{\mathrm{a}}$ \\
\hline $6 / 6$ & $658(47.7 \%)$ & 195 (47.7\%) & Ref. & $88(51.2 \%)$ & Ref. & $37(44.1 \%)$ & Ref. \\
\hline $7 / 6$ & $589(42.7 \%)$ & $173(42.3 \%)$ & $1.01(0.85-1.20)$ & $61(35.5 \%)$ & $1.01(0.79-1.28)$ & $42(50.0 \%)$ & $0.99(0.71-1.40)$ \\
\hline $7 / 7$ & $132(9.6 \%)$ & $41(10.0 \%)$ & $1.02(0.73-1.43)$ & $23(13.4 \%)$ & $1.01(0.63-1.63)$ & $5(5.9 \%)$ & $0.99(0.50-1.96)$ \\
\hline
\end{tabular}

UGT1A $1 * 28$ allele is referred to as 7 , common genotype as 6 .

adjusted for sex and birth decade. 
Table 4. Sex and birth decade adjusted odds ratios (95\% confidence intervals) of pre-B-cell acute lymphoblastic leukemia (ALL), T-cell ALL, and the $t(12 ; 21)$ translocation positive ALL for UGT1A $1 * 28$ genotypes

\begin{tabular}{|c|c|c|c|c|c|c|c|}
\hline \multirow[b]{2}{*}{ Genotype } & \multirow{2}{*}{$\begin{array}{l}\text { Controls } \\
(n=1,379)\end{array}$} & \multicolumn{2}{|c|}{ Pre-B-cell ALL $(n=574)$} & \multicolumn{2}{|c|}{ T-cell ALL $(n=82)$} & \multicolumn{2}{|c|}{$\mathrm{t}(12 ; 21)$ positive $(n=51)$} \\
\hline & & & $\mathrm{OR}^{\mathrm{a}}$ & & $\mathrm{OR}^{\mathrm{a}}$ & & $\mathrm{OR}^{\mathrm{a}}$ \\
\hline $6 / 6$ & $658(47.7 \%)$ & $276(48.1 \%)$ & Ref. & $42(51.1 \%)$ & Ref. & 27 (52.9\%) & Ref. \\
\hline $7 / 7$ & $132(9.6 \%)$ & $57(9.9 \%)$ & $1.00(0.74-1.34)$ & $12(14.6 \%)$ & $1.05(0.53-2.06)$ & $6(11.8 \%)$ & $0.87(0.38-2.07)$ \\
\hline
\end{tabular}

UGT1A $1 * 28$ allele is referred to as 7, common genotype as 6 .

adjusted for sex and birth decade.

the opportunity to test the association between UGT1A1 ${ }^{*} 28$ and ALL in childhood in cases compiled over $30 \mathrm{y}$.

This has led to a reasonably large sample size and a correspondingly high power, reflected in the quite narrow confidence intervals for the primary outcomes.

Case status in the Danish Registry of Childhood Cancer has been meticulously validated and is to the best of our knowledge nationally complete for the study period, making risk of misclassification for both cases and controls negligible.

Of the 903 possible cases, we could not obtain genotypes for 238 (26\%). Reasons for cases missing in the biobank were that parents or patients had chosen the dried blood spot not to be available for research purposes, that initial screening analysis or lather research projects not had left enough material for DNA extraction to be done, or that the dried blood spot was in poor condition. This loss of cases is in all likelihood unrelated to both genotype and outcome, and we do not consider it a potential bias in our estimates.

It is known that $\mathrm{UGT1A} 1^{\star} 28$ genotype frequencies vary with ethnicity (19). However, the Danish population is relatively ethnically homogenous, race is considered of little importance to the incidence of ALL in children, and the observed UGT1A1*28 genotype frequencies correspond well to the expected for a North European population $(18,31)$. Consequently, we do not consider population stratification a potential confounder in our study.

Maternal age, birth order, and birth weight have documented association to ALL in childhood, and are not adjusted for in the logistic regression model. However, as these risk factors expectedly are unrelated to genotype, we do not consider this a potential bias in our estimates.

A limitation in the interpretation of our data is the lack of bilirubin levels for cases and controls and the lack of data on association between UGT1A ${ }^{\star} 28$ genotypes and plasma bilirubin for children after the neonatal period. Associations between plasma bilirubin concentration and UGT1A1*28 genotypes are well established in the adult Danish population (18), and as the UGT1A1*28 allele is a functional polymorphism effect upon UGT1A1 activity seems plausible to be similar for children and adults. However, the effect of altered enzymatic activity on plasma bilirubin concentration could differ between age groups. Hepatic UGT1A1 has marked pleiotropy and metabolizes other endogen and exogenous substances besides bilirubin (19). Although we cannot exclude the existence of a geographically specific UGT1A1 metabolized substance with an impact on ALL in children, no evidence of potential candidates has been published to date. We therefore believe that the external validity of our study is good.

The existence of the Nordic Society for Paediatric Haematology and Oncology database additionally allowed us to do further analysis for a number of subgroups within our primary case population. Analysis was performed on age at onset in three groups to test for the effect modification of age and allow for an induction time of genotype. Separate analysis was also done for pre-B-cell ALL and T-cell ALL as they constitute two distinct forms of childhood ALL and for the $t(12 ; 21)$ positive cases as this chromosomal translocation is most convincingly shown to be congenital in origin $(1,32,33)$.

No association between genotype and outcome was found for any of the secondary outcomes. The smaller numbers of cases in these groups means that the statistical strength is more limited as reflected in wider confidence intervals, but the precision of estimates was still good for most subgroup estimates.

The UGT1A ${ }^{\star} 28$ allele is in the adult one of the strongest available genetic instrumental variables for an endogenous anti-oxidant. Further studies into its potential effects could be relevant for other pediatric out-comes. Such studies could resource effectively be executed within the framework of the Danish Neonatal Screening Biobank, the Danish National Patient Register and several other more specialized diagnostic registers. Bilirubin is highly photosensitive, and cannot be estimated from dried blood spots. Separate studies to explore the relationship between plasma bilirubin concentrations and UGT1A $1^{\star} 28$ genotypes in the pediatric population would there for be relevant.

As umbilical cord concentrations of plasma bilirubin, rather than UGT1A ${ }^{\star} 28$ genotypes, may provide a useful measure of bilirubin exposure in the first days of life this measure should be considered in research aiming to estimate perinatal exposures associations to later outcomes.

In conclusion, we found no association between the UGT1A1*28 genotypes and ALL in children.

\section{METHODS}

\section{Setting}

The study was done in Denmark, which has a public health care system that grants universal access to both primary and specialized care.

\section{Materials}

Our study combined clinical information from The Danish Registry of Childhood Cancer and the registry of the Nordic Society for Paediatric Haematology and Oncology, with genotypes assessed using 
dried blood spot samples stored in the Danish Newborn Screening Biobank.

The Danish Registry of Childhood Cancer, which contains a complete and validated register of all children with ALL in Denmark (34), was used to identify incident cases of ALL cases amongst children 1982-2010. The registry of the Nordic Society for Paediatric Haematology and Oncology encompasses clinical variables on Scandinavian ALL cases since $1982(35,36)$. From this registry data on ALL subtypes (pre-B cell or T cell), $\mathrm{t}(12 ; 21)$ status and age at onset were obtained for cases.

As part of the Danish national neonatal screening for metabolic diseases, a dried blood spot sample is collected from all newborn infants shortly after birth. Since 1982, the excess dried blood spots have been stored in the Danish Neonatal Screening Biobank, which today covers about $99 \%$ of all children born after 1982 (37).

All Danish citizens are at birth assigned a unique 10-digit civil registration number (CPR), which gives information on date of birth and sex and allows unambiguous individual-level identification and data linkage across nationwide public health registers (38).

Our study utilized the CPR number to identify cases in the Danish Newborn Screening Biobank. The controls were selected by taking blood spot samples stored in front of and behind a case in the biobank. As the dried blood spot samples in the biobank are stored chronologically in the original packages from local sample sites, this ensured a frequency match between the case and control populations with regard to time and, to some extent, geography.

From each of the dried blood spot samples two $3.2 \mathrm{~mm}$ disks were punched, DNA extracted, and subsequently whole-genome amplified (wgaDNA) in triplicates, as previously described (39). Genotyping of wgaDNA made from dried blood spot samples has been shown to be feasible and valid (40). Genotyping was performed at LGC Genomics (Teddington, UK) using a custom made Kompetitive Allele Specific PCR genotyping assay. The genotyping laboratory had no knowledge of the identity of either individual samples or the type of project the samples came from.

\section{Statistical Methods}

The STATA statistical software package version 12.1 (StataCorp, College Station, TX) was used for statistical analyses. HardyWeinberg equilibrium for genotype frequencies was explored for controls with the hwsnp command. Associations between genotype and outcome were expressed as ORs using logistic regression with participants with the common genotype as the reference group. Due to the dose-response relationship between UGT1A1 ${ }^{\star} 28$ genotypes and plasma bilirubin, the genotypes were in primary analysis treated as a continuous variable $(0,1$, and 2$)$ and secondarily tested as categorical. Confidence intervals are reported with $95 \%$ limits.

A priori, we decided to control for sex and birth decade as categorical covariates in the logistic models. Testing for a potential effect modulation by covariates was done by fitting interaction terms and inspecting the $P$ values of these for statistical evidence against no interaction, and further by stratifying the analysis on covariates and inspecting results.

Subgroup analysis was done for age at onset in three groups (1-4 y, 5-9 y, and 10-14 y), and for the ALL subtypes: precursor B cell, T cell and $\mathrm{t}(12 ; 21)$ translocation positive status.

\section{Ethics}

The study was approved by the regional Ethics Committees on Human Studies (Jr.nr. M-20110288), the Danish Data Protection Agency (Sagsnr. 1-16-02-238-11), and the Danish Neonatal Biobank Steering Committee and conducted according to the principles of the Declaration of Helsinki. According to Danish law, the regional Ethics Committee can grant exemption from obtaining informed consent for research projects based on biobank material under certain circumstances. For this study, such an exemption was granted.

\section{STATEMENT OF FINANCIAL SUPPORT}

The study was made possible by the grant 2011-45 from the Danish Child Cancer Foundation.

Disclosure: There is no conflict of interest to disclose.

\section{REFERENCES}

1. Greaves MF, Wiemels J. Origins of chromosome translocations in childhood leukaemia. Nat Rev Cancer 2003;3:639-49.

2. Hjalgrim LL, Westergaard T, Rostgaard K, et al. Birth weight as a risk factor for childhood leukemia: a meta-analysis of 18 epidemiologic studies. Am J Epidemiol 2003;158:724-35.

3. Hjalgrim LL, Rostgaard $\mathrm{K}$, Hjalgrim $\mathrm{H}$, et al. Birth weight and risk for childhood leukemia in Denmark, Sweden, Norway, and Iceland. J Natl Cancer Inst 2004;96:1549-56.

4. Naumburg E, Bellocco R, Cnattingius S, Jonzon A, Ekbom A. Supplementary oxygen and risk of childhood lymphatic leukaemia. Acta Paediatr 2002;91:1328-33.

5. Spector LG, Klebanoff MA, Feusner JH, Georgieff MK, Ross JA. Childhood cancer following neonatal oxygen supplementation. J Pediatr 2005;147:2731.

6. Stocker R, Yamamoto Y, McDonagh AF, Glazer AN, Ames BN. Bilirubin is an antioxidant of possible physiological importance. Science 1987;235:1043-6.

7. Llesuy SF, Tomaro ML. Heme oxygenase and oxidative stress. Evidence of involvement of bilirubin as physiological protector against oxidative damage. Biochim Biophys Acta 1994;1223:9-14.

8. Horsfall LJ, Rait G, Walters K, et al. Serum bilirubin and risk of respiratory disease and death. JAMA 2011;305:691-7.

9. Horsfall LJ, Nazareth I, Pereira SP, Petersen I. Gilbert's syndrome and the risk of death: a population-based cohort study. J Gastroenterol Hepatol 2013;28:1643-7.

10. Vitek L, Schwertner HA. The heme catabolic pathway and its protective effects on oxidative stress-mediated diseases. Adv Clin Chem 2007;43: $1-57$.

11. Morris BH, Oh W, Tyson JE, et al.; NICHD Neonatal Research Network. Aggressive vs. conservative phototherapy for infants with extremely low birth weight. N Engl J Med 2008;359:1885-96.

12. Wiedemann M, Kontush A, Finckh B, Hellwege HH, Kohlschütter A. Neonatal blood plasma is less susceptible to oxidation than adult plasma owing to its higher content of bilirubin and lower content of oxidizable Fatty acids. Pediatr Res 2003;53:843-9.

13. Benaron DA, Bowen FW. Variation of initial serum bilirubin rise in newborn infants with type of illness. Lancet 1991;338:78-81.

14. Rodrigues C, Costa E, Vieira E, et al. Bilirubin dependence on UGT1A1 polymorphisms, hemoglobin, fasting time and body mass index. Am J Med Sci 2012;343:114-8.

15. Schwertner HA. Association of smoking and low serum bilirubin antioxidant concentrations. Atherosclerosis 1998;136:383-7.

16. Lawlor DA, Harbord RM, Sterne JA, Timpson N, Davey Smith G. Mendelian randomization: using genes as instruments for making causal inferences in epidemiology. Stat Med 2008;27:1133-63.

17. Bosma PJ, Chowdhury JR, Bakker C, et al. The genetic basis of the reduced expression of bilirubin UDP-glucuronosyltransferase 1 in Gilbert's syndrome. N Engl J Med 1995;333:1171-5.

18. Stender S, Frikke-Schmidt R, Nordestgaard BG, Grande P, Tybjaerg-Hansen A. Genetically elevated bilirubin and risk of ischaemic heart disease: three Mendelian randomization studies and a meta-analysis. J Intern Med 2013;273:59-68.

19. Strassburg CP. Gilbert-Meulengracht's syndrome and pharmacogenetics: is jaundice just the tip of the iceberg? Drug Metab Rev 2010;42: 168-81.

20. Bosma PJ, Seppen J, Goldhoorn B, et al. Bilirubin UDP-glucuronosyltransferase 1 is the only relevant bilirubin glucuronidating isoform in man. J Biol Chem 1994;269:17960-4.

21. Bosma PJ, van der Meer IM, Bakker CT, Hofman A, Paul-Abrahamse M, Witteman JC. UGT1A1 ${ }^{\star} 28$ allele and coronary heart disease: the Rotterdam Study. Clin Chem 2003;49:1180-1.

22. Jirásková A, Novotný J, Novotný L, et al. Association of serum bilirubin and promoter variations in HMOX1 and UGT1A1 genes with sporadic colorectal cancer. Int J Cancer 2012;131:1549-55.

23. de Vries HS, Te Morsche RH, Jenniskens K, Peters WH, de Jong DJ. A functional polymorphism in UGT1A1 related to hyperbilirubinemia 
is associated with a decreased risk for Crohn's disease. J Crohns Colitis 2012;6:597-602.

24. Schwertner HA, Vítek L. Gilbert syndrome, UGT1A1²8 allele, and cardiovascular disease risk: possible protective effects and therapeutic applications of bilirubin. Atherosclerosis 2008;198:1-11.

25. Coughtrie MW, Burchell B, Leakey JE, Hume R. The inadequacy of perinatal glucuronidation: immunoblot analysis of the developmental expression of individual UDP-glucuronosyltransferase isoenzymes in rat and human liver microsomes. Mol Pharmacol 1988;34:729-35.

26. Kawade N, Onishi S. The prenatal and postnatal development of UDPglucuronyltransferase activity towards bilirubin and the effect of premature birth on this activity in the human liver. Biochem J 1981;196:257-60.

27. Watchko JF, Lin Z, Clark RH, Kelleher AS, Walker MW, Spitzer AR; Pediatrix Hyperbilirubinemia Study Group. Complex multifactorial nature of significant hyperbilirubinemia in neonates. Pediatrics 2009;124:e868-77.

28. Kaplan M, Hammerman C, Renbaum P, Klein G, Levy-Lahad E. Gilbert's syndrome and hyperbilirubinaemia in ABO-incompatible neonates. Lancet 2000;356:652-3.

29. Li J, Cnattingus S, Gissler M, et al. The 5-minute Apgar score as a predictor of childhood cancer: a population-based cohort study in five million children. BMJ Open 2012;2:e001095.

30. Zhu J, Xu Y, Zhang G, Wu M, Du L. Total serum bilirubin level in umbilical cord blood and respiratory distress syndrome in very low birth weight infants. J Perinat Med 2011;40:91-95.

31. Ekblom K, Marklund SL, Jansson JH, et al. Plasma bilirubin and UGT1A1 ${ }^{\star} 28$ are not protective factors against first-time myocardial infarction in a prospective, nested case-referent setting. Circ Cardiovasc Genet 2010;3:340-7.
32. McHale CM, Wiemels JL, Zhang L, et al. Prenatal origin of TEL-AML1positive acute lymphoblastic leukemia in children born in California. Genes Chromosomes Cancer 2003;37:36-43.

33. Taub JW, Konrad MA, Ge Y, et al. High frequency of leukemic clones in newborn screening blood samples of children with B-precursor acute lymphoblastic leukemia. Blood 2002;99:2992-6.

34. Schrøder H, Johnsen SP, Larsson H, Rechnitzer C, Wehner PS, Rosthøj S. [The Danish Registry of Childhood Cancer]. Ugeskr Laeg 2012;174:2542.

35. Gustafsson G, Garwicz S, Hertz H, et al. A population-based study of childhood acute lymphoblastic leukemia diagnosed from July 1981 through June 1985 in the five Nordic countries. Incidence, patient characteristics and treatment results. Acta Paediatr Scand 1987;76:781-8.

36. Schmiegelow K, Forestier E, Hellebostad M, et al.; Nordic Society of Paediatric Haematology and Oncology. Long-term results of NOPHO ALL-92 and ALL-2000 studies of childhood acute lymphoblastic leukemia. Leukemia 2010;24:345-54.

37. Nørgaard-Pedersen B, Hougaard DM. Storage policies and use of the Danish Newborn Screening Biobank. J Inherit Metab Dis 2007;30:530-6.

38. Pedersen CB, Gøtzsche H, Møller JO, Mortensen PB. The Danish Civil Registration System. A cohort of eight million persons. Dan Med Bull 2006;53:441-9.

39. Hollegaard MV, Thorsen P, Norgaard-Pedersen B, Hougaard DM. Genotyping whole-genome-amplified DNA from 3- to 25-year-old neonatal dried blood spot samples with reference to fresh genomic DNA. Electrophoresis 2009;30:2532-5.

40. Hollegaard MV, Grauholm J, Børglum A, et al. Genome-wide scans using archived neonatal dried blood spot samples. BMC Genomics 2009; 10:297. 